\title{
Endogenous Cushing's syndrome: The Philippine general hospital experience
}

\author{
Tom Edward N. Lo, Joyce M. Cabradilla, Sue Ann Lim, Cecilia A. Jimeno \\ Section of Endocrinology and Metabolism, Department of Medicine, University of the Philippines-Philippine General \\ Hospital, Philippines
}

Correspondence: Tom Edward N. Lo. Address: 668 Quirino Avenue Jaleville Subdivision Kabihasnan, Paranaque, Philippines. Email: tomedwardlo@yahoo.com

Received: September 28, 2013 Accepted: December 23, 2013

Online Published: December 29, 2013

DOI : $10.5430 /$ jst.v4n1p1

URL: http://dx.doi.org/10.5430/jst.v4n1p1

\begin{abstract}
Background and significance: Endogenous Cushing's syndrome is a rare disease entity approximately 13 cases per million population. In Asia, and specifically in the Philippines, the exact incidence is unknown. As well, it's clinical presentation among Filipinos is not well described. The local epidemiology of obesity, diabetes and hypertension are different from other countries. These conditions are the usual initial differential diagnosis for Cushing's syndrome. This case collection is thus important for the awareness of clinicians on the clinical presentation and course of individuals with this condition.
\end{abstract}

Methodology: Medical records of patients diagnosed with Endogenous Cushing's Syndrome (non steroid-induced) within the year 2005-2011 were retrieved using the ICD 10 code for Cushing's Syndrome (E24). Exogenous Cushing's Syndrome (steroid-induced) were all excluded from the study. All selected medical records were individually reviewed, assessed and carefully recorded.

Results: The study included 19 cases (8 pituitary adenoma, 7 adrenal adenoma, 4 ectopic adenoma) of endogenous Cushing's Syndrome. Female predominance was noted. The most commonly reported clinical symptom and sign were weight gain and moon facies respectively. Short clinical course, hyperpigmentation, ecchymoses, behavioural changes and severe hypokalemia were observed to be more prominent among cases with ectopic sources. All cases had significantly elevated midnight serum cortisol level and 24 hour urine cortisol with ectopic sources exhibiting the highest level. There was no observed correlation between the tumour size and level of serum cortisol in all types. Majority underwent tumour resection leading to complete reversal of steroid excess.

Conclusion: In our series of 19 cases, adrenal, pituitary and ectopic foci were the main sources of endogenous steroid excess. Ectopic ACTH syndrome manifested differently with other causes providing clues that might help in rapid clinical differentiation. Imaging studies should only be used to confirm tumour location after rigorous biochemical tests. Surgical management remained to be the definitive cure for most of the cases, hence prompt diagnosis and localization is necessary.

\section{Key words}

Cushing's syndrome, Pituitary adenoma, Cushing's Disease, Adrenal adenoma, Ectopic ACTH-producing tumor 


\section{Introduction}

\subsection{Background}

Cushing's syndrome is a hormonal disorder brought about by chronic exposure of body's tissues to elevated levels of steroid (cortisol) leading to typical signs and symptoms of truncal obesity, moon facies, easy bruisability and poor wound healing, osteoporosis, hirsutism and menstrual irregularities in women, and hypogonadism in men. Other non-specific signs and symptoms like fatigue, muscle weakness, hypertension, hyperglycemia, polyuria, polydipsia, irritability, anxiety, depression and abnormal body fat depositions are also observed. Cushing's syndrome is relatively rare and most commonly affects adults aged 20 to 50. People with this disorder have an increased risk of developing obesity, hypertension and diabetes ${ }^{[1]}$.

Majority of cases are due to exogenous intake of glucocorticoids (prednisone) for treatment of other medical conditions like asthma, rheumatoid arthritis, lupus, and other inflammatory diseases. While the less common endogenous Cushing syndrome has an annual incidence estimated at 13 cases per million individuals. Of these cases, approximately $70 \%$ are due to Cushing disease, that is, a pituitary ACTH-producing tumor; 15\% to ectopic Adrenocorticotropic Hormone (ACTH); and $15 \%$ to a primary adrenal tumor ${ }^{[2]}$.

Pituitary adenomas (Cushing's Disease) are the most common cause of Endogenous Cushing's syndrome. These benign, or noncancerous, tumors of the pituitary gland secrete extra ACTH. Most people with the disorder have a single adenoma ${ }^{[3]}$. Ectopic ACTH Syndrome is due to an overproduction of ACTH outside the Hypothalamo-Pituitary-Adrenal axis leading to features of Cushing's. Lung tumors (small cell type) cause more than half of these cases. In rare instances, an abnormality of the adrenal glands, most often an adrenal adenoma, causes Cushing's syndrome. Adrenocortical carcinomas are the least common cause of Cushing's syndrome but commonly results to very high hormone levels and rapid development of symptoms leading to deterioration ${ }^{[1]}$.

\subsection{Statement of the problem}

Endogenous Cushing's Syndrome cases are indeed rarely seen by common physicians leading to delay, misdiagnosis and even under-management resulting to higher mortality rates. Majority of cases are often with causes that are fully reversible once detected early. Due to limited amount of knowledge and studies published regarding these cases, physicians or even specialists usually have a hard time recognizing or diagnosing clinical pearls, clues or subtle signs of the disease. Confusion with tumor localization adds to its clinical challenge. In the Philippines where there is limited resources and access to information, it is difficult to rapidly assess and diagnose such patients. Recent published studies are mostly derived from literatures abroad and there are only a few studies done in our country. Only one local study from the Philippine General Hospital (PGH) in year 2000 was done and published. No follow-up studies were done since then. With this in mind, it is quite significant for a local study to be done that will perhaps show the description and causes of endogenous Cushing's in the Philippines.

\subsection{Objectives}

1) To describe the socio-demographic profile, clinical signs and symptoms, associated co-morbidities, biochemical and radiologic features and final histopathologic findings of patients with Endogenous Cushing’s Syndrome (non steroid-induced).

2) To identify, compare and contrast the different types of Endogenous Cushing’s Syndrome.

3) To note the treatment outcome and prognosis of all included cases. 


\subsection{Significance of the study}

Because of its rare incidence and limited number of published researches locally, this study is a significant source of information for current medical practitioners who encounter patients with endogenous Cushing's syndrome. Our collection of cases adds to the clinical knowledge regarding the disease especially because it is an uncommon illness. Local data on the demographic profile and clinical presentation provides Filipino physicians clues in recognizing possible cases for screening. Morbidities and mortalities were documented such that their presence might inform and help physicians in their clinical practice. This description and collection of cases will serve as a template for future database for this rare yet interesting disease.

\section{Materials and methods}

\subsection{Case retrieval}

Medical records of patients diagnosed with Cushing's Syndrome seen in the Philippine General Hospital, within the year 2005- 2011, were retrieved using 3 methods. Cases were confined in the adult population with age $>19$ years. First we utilized the ICD 10 code for Cushing's Syndrome (E24). Second method used retrieval of the in-patient referral logbook of the section of Endocrinology. Third was retrieval of the patient's logbook at the General Endocrinology Clinic. Patient's case numbers were all listed and forwarded to the medical records section for chart retrieval.

\subsection{Selection of medical cases}

All selected and retrieved medical records were individually reviewed and assessed for eligibility. Cushing's Syndrome due to exogenous steroid intake were automatically excluded in the study. The diagnosis of Cushing's Syndrome was based on clinical features associated with hypercortisolemia, an elevated midnight sleeping cortisol level, and lack of suppression of serum cortisol after an oral 2-mg/d 48-h low-dose dexamethasone suppression test. Medical charts that fulfilled the clinical diagnosis of an endogenous Cushing's Syndrome were included in the study.

\subsection{Data processing}

General profile of all patients obtained from the clinical records such as age, sex and race were tabulated and synthesized to generate the demographic profile of the sample. Chief complaints, signs, symptoms and physical examination findings were summarized. Laboratory and diagnostic tests done in all patients were carefully reviewed and analyzed. Tumor sizes noted from imaging studies were recorded and the greatest diameter noted was obtained for analysis. Outcomes and histo-pathologic diagnoses were taken into consideration. In-patient causes of mortality and morbidity were also investigated.

\section{Results}

\subsection{Demographic data}

Of the 144 charts listed with Cushing's Syndrome as a diagnosis, only 103 charts (71.5\%) were retrieved. After thorough chart review, only 19 cases were included. Majority were found to have exogenous steroid administration hence were excluded from this study. ACTH-producing pituitary adenoma or Cushing's Disease represented $42 \%$ of the cases (8 cases). Thirty-six percent were Cortisol-producing Adrenal Adenoma (7 cases) while only 21\% (4 cases) were considered ectopic ACTH-producing tumor. Lungs, pancreas and thymus were the identified ectopic sources of steroid excess.

Ages range from 17-54 years old. Median age was 26. Pituitary cases were younger at the time of diagnosis while Ectopic cases had the widest and most varied age. Female to male ratio was 8.5:1. Overall disease duration was 2.3 years + SD 3 
years. Ectopic cases had the shortest disease duration ( 0.83 years + SD 0.81$)$ followed by the Adrenal cases (1.6 years + SD 2.4) and pituitary cases (3.6 years + SD 3.6). Hypertension was seen in majority of cases. Mortality rates were generally low except in patients found to have ectopic sources (Table 1).

Table 1. Distribution of patients with endogenous Cushing's syndrome according to demographic and clinical characteristics

\begin{tabular}{lllll}
\hline & Endogenous Cushing's & \multicolumn{3}{c}{ Type } \\
\cline { 3 - 5 } Baseline characteristics & $\mathbf{n} \mathbf{1 9}(\mathbf{\%})$ & $\begin{array}{l}\text { Pituitary } \\
\mathbf{n} / \mathbf{8}(\%)\end{array}$ & $\begin{array}{l}\text { Adrenal } \\
\mathbf{n} / \mathbf{7}(\%)\end{array}$ & $\begin{array}{l}\text { Ectopic } \\
\mathbf{n} / \mathbf{4}(\%)\end{array}$ \\
\hline Female Percentage & $17 / 19(89.5)$ & $7 / 8(87.5)$ & $7 / 7(100)$ & $3 / 4(75)$ \\
Disease Duration (yr) & $2.3 \pm 3$ & $3.6 \pm 3.6$ & $1.6 \pm 2.4$ & $0.83 \pm 0.81$ \\
Hypertension & $16 / 19(84.2)$ & $6 / 8(75)$ & $6 / 7(85.7)$ & $4 / 4(100)$ \\
Diabetes & $5 / 19(26.3)$ & $2 / 8(25)$ & $2 / 7(28.6)$ & $1 / 4(25)$ \\
Mortalities & $4 / 19(21.1)$ & $0 / 8(0)$ & $1 / 7(14.3)$ & $3 / 4(75)$ \\
\hline
\end{tabular}

\subsection{Clinical signs and symptoms}

The most commonly reported clinical symptoms in patients with endogenous Cushing's Syndrome were weight gain (94.7\%), menstrual irregularities (70.6\%) and weakness (63.2\%). Aside from the 3 common symptoms, majority of patients with Cushing's Disease also had easy fatigability (63.2\%). The adrenal adenoma cases also presented with weight gain and weakness, but most also had easy brusability (57.1\%) and headache (42.9\%). All cases of ectopic ACTH producing tumors presented with the 3 most common symptoms. Aside from that, prominent symptoms were also easy fatigability, proximal myopaty and easy bruisability (75\%).

Moon facies and buffalo hump were the most common signs observed and were present in all cases. Other common signs noted in pituitary cases were abdominal striae (79\%) and truncal obesity (73.7\%). Cases of adrenal adenoma also had prominent edema (85.7\%) and plethora (74.1\%). Aside from moon facies and buffalo hump, all cases of ectopic ACTH producing producing tumors also presented with hyperpigmentation, truncal obesity and abdominal striae (Table 2).

\subsection{Biochemical and radiologic features}

Following the algorithm for patients with suspected Cushing's Syndrome, all cases were confirmed with 24-hour urinary free cortisol excretion and dexamethasone overnight test (Figure 1). Considered as an evidence of steroid excess and used for the diagnosis of Cushing's Syndrome, all cases had significantly elevated midnight serum cortisol level (Mean of 1,028 $\mathrm{nmol} / \mathrm{l}$ ) and 24 hour urine cortisol (Mean of $743.9 \mu \mathrm{g} / 24 \mathrm{hr}$ ). Ectopic sources exhibited significantly higher level of steroid excess (Mean Serum Cortisol of 2,091.8 nmol/l and Mean 24hr urine Cortisol of 1,505 $\mu \mathrm{g} / 24 \mathrm{hr}$ ). Serum potassium was also noted to be significantly lower among cases with ectopic sources. Serum ACTH levels were elevated in both pituitary and ectopic sources as opposed to adrenal sources which showed significantly lower values. Serum ACTH levels were significantly higher in patients with ectopic sources reflecting severe steroid excess (Table 3 and Figure 2).

Midnight serum cortisol, serum ACTH, serum potassium and low dose dexamethasone suppression tests were done in all cases. Only 10 (52.6\%) cases proceeded with high dose dexamethasone suppression test. Most of which were used in patients suspected to have pituitary and ectopic sources. More than $90 \%$ of the cases showed unsuppressed serum cortisol 
level after low dose dexamethasone suppression test. Only ectopic sources showed unsuppressed serum cortisol level after high dose dexamethasone suppression test. More than half of the cases presented with significant dyslipidemia.

Table 2. Clinical features of patients with endogenous Cushing's syndrome

\begin{tabular}{|c|c|c|c|c|}
\hline \multirow{2}{*}{ Clinical features } & \multirow{2}{*}{$\begin{array}{l}\text { Endogenous } \\
\text { Cushing's } \\
\text { n/19 (\%) }\end{array}$} & \multicolumn{3}{|c|}{ Type } \\
\hline & & $\begin{array}{l}\text { Pituitary } \\
\text { n/8 (\%) }\end{array}$ & $\begin{array}{l}\text { Adrenal } \\
\text { n/7 (\%) }\end{array}$ & $\begin{array}{l}\text { Ectopic } \\
\text { n/4 (\%) }\end{array}$ \\
\hline \multicolumn{5}{|l|}{ Symptoms } \\
\hline Easy Fatigability & 10/19 (52.6) & $5 / 8(62.5)$ & $2 / 7(28.6)$ & $3 / 4(75)$ \\
\hline Weakness & 12/19 (63.2) & 5/8 (62.5) & 3/7 (42.9) & 4/4 (100) \\
\hline Proximal Myopathy & 7/19 (36.8) & 2/8 (25.0) & 2/7 (28.6) & 3/4 (75) \\
\hline Headache & 7/19 (36.8) & $3 / 8(37.5)$ & 3/7 (42.9) & $1 / 4(25)$ \\
\hline Dizziness & 3/19 (15.8) & 1/8 (12.5) & 1/7 (14.3) & 1/4 (25) \\
\hline Menstrual Irregularity ${ }^{*}$ & $12 / 17(70.6)$ & $7 / 7(100)$ & 2/7 (28.6) & $3 / 3(100)$ \\
\hline Behavioral Changes & 3/19 (15.8) & 1/8 (12.5) & $0 / 7(0)$ & 2/4 (50) \\
\hline Easy Bruisability & 10/19 (52.6) & 3/8 (37.5) & 4/7 (57.1) & $3 / 4(75)$ \\
\hline Visual Changes & 4/19 (21.1) & 2/8 (25) & 2/7 (28.6) & $0 / 4(0)$ \\
\hline Weight Gain & 18/19 (94.7) & 8/8 (100) & 6/7 (85.7) & 4/4 (100) \\
\hline 3Ps (Polydipsia, Polyuria, Polyphagia) & 3/19 (15.8) & 2/8 (25) & $0 / 7(0)$ & $1 / 4(25)$ \\
\hline \multicolumn{5}{|l|}{ Signs } \\
\hline Moon Facies & 19/19 (100) & 8/8 (100) & 7/7 (100) & 4/4 (100) \\
\hline Supraclavicular Fat Pads & $13 / 19(68.4)$ & $5 / 8(62.5)$ & $5 / 7(71.4)$ & $3 / 4(75)$ \\
\hline Buffalo Hump & 19/19 (100) & 8/8 (100) & $7 / 7(100)$ & $4 / 4(100)$ \\
\hline Plethora & 10/19 (52.6) & 3/8 (37.5) & $5 / 7(71.4)$ & $2 / 4(50)$ \\
\hline Acne Formation & 9/19 (47.4) & $5 / 8(62.5)$ & 2/7 (28.6) & $2 / 4(50)$ \\
\hline Hyperpigmentation & 6/19 (31.6) & 2/8 (25) & $0 / 7(0)$ & 4/4 (100) \\
\hline Truncal Obesity & $14 / 19(73.7)$ & 6/8 (75) & $4 / 7(57.1)$ & $4 / 4(100)$ \\
\hline Hirsutism & $11 / 19(57.9)$ & $5 / 8(62.5)$ & $4 / 7(57.1)$ & $2 / 4(50)$ \\
\hline Abdominal Striae & 16/19 (79.0) & 7/8 (87.5) & 4/7 (57.1) & 4/4 (100) \\
\hline Edema & 10/19 (52.6) & 2/8 (25) & 6/7 (85.7) & 2/4 (50) \\
\hline Ecchymoses & 8/19 (42.1) & 4/8 (50) & 1/7 (14.3) & 3/4 (75) \\
\hline
\end{tabular}

${ }^{*}$ n females only 
Ultrasound, CT scan and MRI were the usual radiologic tests done to confirm and localize the exact source of steroid excess in all cases. The choice of imaging study depended on the suspected source such that majority of patients with pituitary sources underwent pituitary MRI while patients with adrenal sources had Abdominal CT scan. Ectopic sources utilized all of the imaging modalities to accurately detect the source of steroid excess. Some ectopic sources were discovered on autopsy findings after death from complications. Pituitary sources exhibited the smallest tumor sizes ranging from $0.3 \mathrm{~cm}$ to $1.9 \mathrm{~cm}$ while adrenal sources manifested with larger sizes ranging from $2.5 \mathrm{~cm}$ to $15 \mathrm{~cm}$. Small cell lung cancer, pancreatic head tumor with liver metastases and thymoma were some of the ectopic sources identified in this study. There was no observed correlation between the tumor size and level of serum cortisol in all types of endogenous Cushing's Syndrome (Table 4).

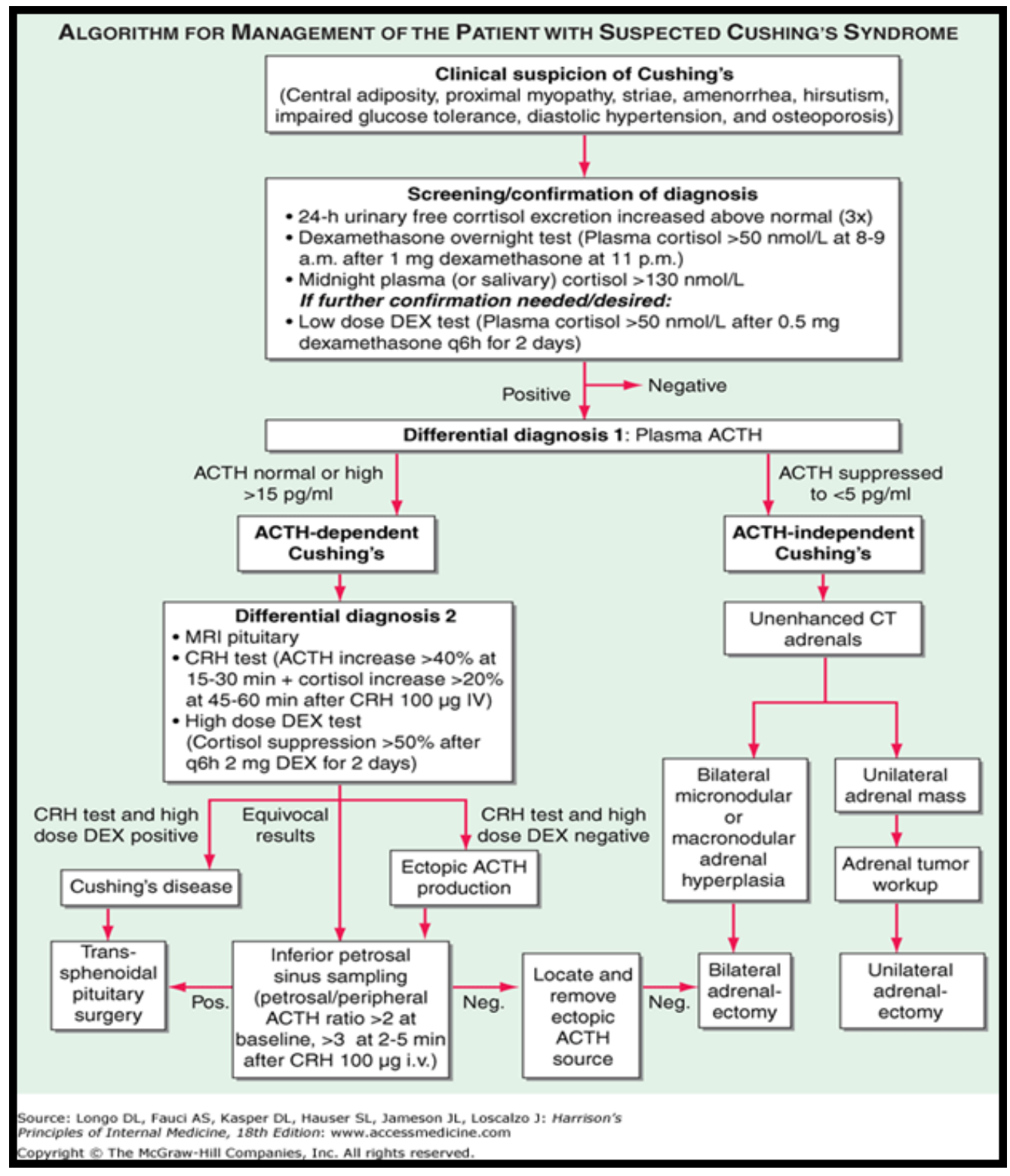

Figure 1. Algorithm for the management of patients suspected with Cushing's syndrome

Majority of patients underwent tumor resection leading to clinical improvement and complete reversal of steroid excess. Cushing's disease had the best prognosis with no reported mortalities. Only 1 patient with adrenal tumor died due to complications of pregnancy and delayed surgical management. Three out of 4 patients with ectopic ACTH syndrome died 
from significant complications (infection, hyperglycemia) brought about by severe steroid excess. Short and rapidly progressing clinical course prevented from effectively localizing the source of hormone excess. Ectopic foci were only determined post mortem in 2 patients (Table 1).

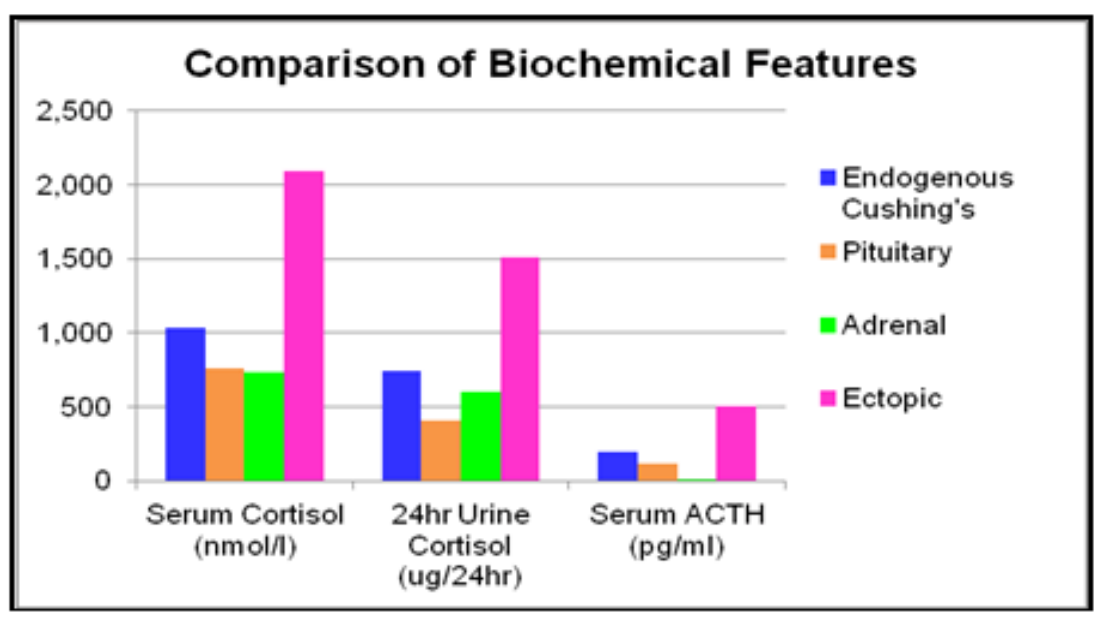

Figure 2. Comparison of biochemical features among different sources of endogenous Cushing’s syndrome

Table 3. Biochemical features of patients with endogenous Cushings syndrome

\begin{tabular}{|c|c|c|c|c|}
\hline \multirow{2}{*}{ Biochemical features } & \multirow{2}{*}{$\begin{array}{l}\text { Endogenous } \\
\text { Cushing's }\end{array}$} & \multicolumn{3}{|c|}{ Type } \\
\hline & & Pituitary & Adrenal & Ectopic \\
\hline Serum Cortisol (nmol/l) & $1,028.3(n=19)$ & $757.8(\mathrm{n}=8)$ & $729.9(\mathrm{n}=7)$ & $2091.8(n=4)$ \\
\hline 24hr Urine Cortisol ( $\mu \mathrm{g} / 24 \mathrm{hr})$ & $743.9(n=17)$ & $406.8(n=6)$ & $598(n=7)$ & $1505.0(n=4)$ \\
\hline Serum ACTH (pg/ml) & $192.4(n=19)$ & $115.1(n=8)$ & $4.5(n=7)$ & $505.8(n=4)$ \\
\hline Serum Potassium (mmol/l) & $3.29(n=19)$ & $3.75(n=8)$ & $3.37(n=7)$ & $2.23(n=4)$ \\
\hline $\begin{array}{l}\text { Patients with Unsuppressed Low Dose Dexa } \\
\text { Suppression Test (\%) }\end{array}$ & $94.4(n=18)$ & $85.7(n=7)$ & $100.0(n=7)$ & $100.0(n=4)$ \\
\hline $\begin{array}{l}\text { Patients with Unsuppressed High Dose Dexa } \\
\text { Suppression Test (\%) }\end{array}$ & $50.0(n=10)$ & $0.0(n=5)$ & $100.0(n=1)$ & $100.0(n=4)$ \\
\hline Patients with Dyslipidemia (\%) & $68.4(n=19)$ & $75.0(n=8)$ & $42.9(n=7)$ & $100.0(n=4)$ \\
\hline
\end{tabular}

\section{Discussion}

This retrospective study of 19 cases presented the 3 main causes of Endogenous Cushing's Syndrome namely pituitary, adrenal and ectopic sources. ACTH-producing Pituitary Adenoma appeared to be the most common cause which is consistent with the usual etiologies of endogenous Cushing's worldwide. For an unknown reason, females remained to be more commonly affected with Cushing's Disease and Adrenal Cushing's. Although Ectopic sources are usually more prevalent among males, our study showed female predominance (3 out of 4 cases). All of which presented with aggressive malignancies leading to eventual demise. Only 1 male patient exhibited ectopic ACTH syndrome from a benign thymoma which resulted into significant resolution of the disease after surgical tumor removal. 
There were 4 cases of ectopic ACTH-producing tumor. Sources were one from the lungs, 2 cases from the pancreas and 1 case from the thymus. Comparing to international data, more than half of the tumors causing ectopic ACTH syndrome were found in the lungs (bronchial carcinoid tumours, small cell carcinoma or adenocarcinomas) or in the thymus. Only $1 / 3$ of primary tumours were found in the abdomen, most frequently in the pancreas or the colon ${ }^{[4]}$.

Table 4. Radiologic and biochemical correlation of patients with endogenous Cushing’s syndrome

\begin{tabular}{|c|c|c|c|c|c|c|}
\hline Case & Ultrasound & CT scan & MRI & Tumor size (cm) & $\begin{array}{l}\text { Serum Cortisol } \\
(\mathrm{nmol} / \mathrm{l})\end{array}$ & Histopath result \\
\hline 1 & $(-)$ & $(-)$ & $(+)$ & 0.3 & 1,105 & Pituitary Adenoma \\
\hline 2 & $(+)$ & $(-)$ & $(+)$ & 0.6 & 845 & Pituitary Adenoma \\
\hline 3 & $(-)$ & $(-)$ & $(+)$ & 1.8 & 554 & Pituitary Adenoma \\
\hline 4 & $(-)$ & $(-)$ & $(+)$ & 1.3 & 363 & Pituitary Adenoma \\
\hline 5 & $(+)$ & $(+)$ & $(-)$ & 1.4 & 1,313 & Pituitary Adenoma \\
\hline 6 & $(-)$ & $(-)$ & $(+)$ & 0.3 & 585 & Pituitary Adenoma \\
\hline 7 & $(-)$ & $(-)$ & $(+)$ & 3.0 & 432 & Pituitary Adenoma \\
\hline 8 & $(-)$ & $(-)$ & $(+)$ & 1.9 & 865 & Pituitary Adenoma \\
\hline 9 & $(-)$ & $(+)$ & $(+)$ & 8.0 & 780 & Adrenal Adenoma \\
\hline 10 & $(+)$ & $(+)$ & $(-)$ & 15.0 & 614 & Adrenal Adenoma \\
\hline 11 & $(+)$ & $(+)$ & $(+)$ & 2.5 & 579 & Adrenal Adenoma \\
\hline 12 & $(-)$ & $(+)$ & $(-)$ & 3.1 & 612 & Adrenal Adenoma \\
\hline 13 & $(+)$ & $(+)$ & $(-)$ & 3.6 & 1,344 & Adrenal Adenoma \\
\hline 14 & $(-)$ & $(-)$ & $(+)$ & 3.0 & 637 & Adrenal Adenoma \\
\hline 15 & $(+)$ & $(+)$ & $(-)$ & 3.2 & 543 & Adrenal Adenoma \\
\hline 16 & $(+)$ & $(+)$ & $(-)$ & 2.5 & 1,583 & Small Cell Lung CA \\
\hline 17 & $(+)$ & $(+)$ & $(-)$ & 1.3 & 1,125 & $\begin{array}{l}\text { ACTH-producing Pancreatic } \\
\text { Tumor with Liver Metastases }\end{array}$ \\
\hline 18 & $(+)$ & $(+)$ & $(-)$ & 3.0 & 4,863 & $\begin{array}{l}\text { ACTH-producing Pancreatic } \\
\text { Tumor with Liver Metastases }\end{array}$ \\
\hline 19 & $(+)$ & $(+)$ & $(-)$ & 4.0 & 796 & ACTH-producing Thymoma \\
\hline
\end{tabular}

Evidence of steroid excess as manifested by various clinical signs, symptoms and biochemical features were consistently seen in all our cases. The overall clinical features noted in our study were similar to the previous local Philippine study made in $2000{ }^{[5]}$. Patients with ectopic ACTH syndromes were found to have more severe and more prominent clinical 
features suggesting higher steroid excess leading to greater complications and worse mortality rates, which was seen in this study. Hyperpigmentation was seen more exclusively among patients with ectopic ACTH. Significant hypokalemia leading to proximal myopathy was also prominent among patients with ectopic ACTH. These peculiar findings might provide physicians with better clinical clues for earlier diagnosis and prompt management.

Visual changes (Bitemporal Hemianopsia) might not be commonly observed in pituitary adenoma because of the prominence of endocrine abnormalities despite small tumor size. Serum ACTH effectively differentiated ACTHdependent (Pituitary and Ectopic Source) and ACTH-independent (Adrenal Source) tumors in our study. Most cases of ectopic ACTH syndrome had rapid short courses especially those associated with malignancies of the lungs and pancreas. These findings were all consistently seen in our ectopic ACTH cases. Several other studies done in India and Brazil all showed similarities with our ectopic ACTH cases $^{[6,7]}$.

The three most useful screening tests for the diagnosis of Cushing's Syndrome (midnight serum cortisol, 24 hour urine cortisol and low dose dexamethasone suppression test) were done in all cases to establish the presence of steroid excess in patients suspected to have endogenous Cushing's syndrome. High dose dexamethasone suppression test effectively differentiated pituitary from ectopic sources in our study, although it was seldom utilized. MRI is the mainstay of pituitary assessment in Cushing's disease ${ }^{[8]}$. This was seldomly used in the presented cases.

Investigation of patients with 'occult' ectopic ACTH production represents a major challenge since clinical, biochemical and radiological features are often indistinguishable from Cushing's disease ${ }^{[9]}$. CT of the chest, abdomen and pelvis with intravenous injection of contrast medium is the most sensitive imaging modality for the identification of the ectopic source although no single imaging modality can itself identify and localize every tumor responsible for ectopic ACTH syndrome ${ }^{[10]}$. In this study, the case of ectopic ACTH tumor from the lungs was easily clinched with the use of the chest x-ray. Of the 2 cases of pancreatic tumors, only 1 was diagnosed using abdominal CT scan and the other was diagnosed post-portem from autopsy. There was a very thorough investigation for the thymoma case and all basic imaging tests were done.

Selective tumor resection through specific surgical procedure remains to be the treatment of choice for most endogenous Cushing's Syndrome. Most of the pituitary and adrenal cases included in this study underwent curative surgical procedures leading to complete reversal of steroid excess. Unfortunately, most of the Ectopic ACTH syndrome cases in this study died of serious complications before effective localization and surgical therapy can be offered.

\section{Conclusion}

Adrenal, pituitary and ectopic foci are the main sources of endogenous steroid excess. Ectopic ACTH syndrome manifests differently with other causes providing clinical clues that might help in rapid clinical differentiation. Imaging studies should only be used to confirm tumour location after rigorous biochemical tests. Surgical management remains to be the definitive cure for most of the cases, hence prompt diagnosis and localization is necessary.

\section{References}

[1] Govindan R, Page N, Morgensztern D, et al. Changing epidemiology of small-cell lung cancer in the United States over the last 30 years: analysis of the surveillance, epidemiologic, and end results database. Journal of Clinical Oncology. 2006; 24: $4539-4544$. PMid:17008692 http://dx.doi.org/10.1200/JCO.2005.04.4859

[2] eMedicine[Internet]. Adler, GK. Cushing’s Syndrome. [Cited October 2009]. Available from: http://emedicine.medscape.com/article/117365-overview.

[3] Nieman LK, Ilias I. Evaluation and treatment of Cushing's syndrome. The Journal of American Medicine. 2005;118(12):1340-1346. PMid:16378774 http://dx.doi.org/10.1016/j.amjmed.2005.01.059

[4] Isidori AM, Lenzi A. Ectopic ACTH Syndrome. Arq Bras Endocrinology Metabolism. 2007; 51(8): 1217-1225.

Published by Sciedu Press 
http://dx.doi.org/10.1590/S0004-27302007000800007

[5] Montecillo FMP, Trajano-Acampado, L. Clinical Profile of Patients with Cushing's Syndrome seen at the UP-PGH: a five-year Review. Philippine Journal of Internal Medicine. 2004 March; 42: 65-68.

[6] Salgado LR, et al. Ectopic ACTH Syndrome: our Experience with 25 cases. European Journal of Endocrionlogy. 2006; 155: 725-733. PMid:17062889 http://dx.doi.org/10.1530/eje.1.02278

[7] Bhansali A. et al. Ectopic Cushing’s Syndrome: Experience from a tertiary Care Centre. Indian Journal of Medical Researches. January. 2009; 129: 33-41.

[8] Woo YS, Isidori AM, Wat WZ, Kaltsas GA, Afshar F, Sabin I, et al. Clinical and biochemical characteristics of adrenocorticotropin-secreting macroadenomas. Journal of Clinical Endocrinology and Metabolism. 2005; 90(8): 4963-9. PMid:15886242 http://dx.doi.org/10.1210/jc.2005-0070

[9] Vincent JM, Trainer PJ, Reznek RH, Marcus AJ, Dacie JE, Armstrong P, et al. The radiological investigation of occult ectopic ACTH-dependent Cushing's syndrome. Clinical Radiology. 1993; 48(1): 11-7. http://dx.doi.org/10.1016/S0009-9260(05)80100-X

[10] Isidori AM, et.al. The Ectopic Adrenocorticotropin Syndrome: Clinical Features, Diagnosis, Management and Long-term Follow up. The Journal of Clinical Endocrinology and Metabolism. 2006; 91(2): 371-377. PMid:16303835

http://dx.doi.org/10.1210/jc.2005-1542 